\title{
DIFFUSE CLUSTER RADIO SOURCES
}

\author{
L. FERETTI AND G. GIOVANNINI
}

Istituto di Radioastronomia CNR, Bologna, Italy

Dipartimento di Astronomia dell'Università, Bologna, Italy

\section{Introduction}

Diffuse radio sources in clusters remain a poorly understood phenomenon. They are very extended sources $(0.4-0.6 \mathrm{Mpc})$, of low surface brightness and steep spectrum, which cannot be identified with any active radio galaxy. They are a rare phenomenon, as they have been found so far in few clusters of galaxies. This paper reviews the current findings about this kind of sources, and the suggestions about their formation and evolution.

A Hubble constant $\mathrm{H}_{0}=100 \mathrm{~km} / \mathrm{s} \mathrm{Mpc}$ is used throughout.

In the effort of investigating the properties of diffuse cluster sources, we classify them in three classes: cluster-wide halos, relics and mini-halos. Cluster-wide halos include sources located at the cluster centers, while relics are those diffuse extended sources located at the cluster peripheries. Moreover, in some clusters with a central dominant galaxy, the relativistic particles can be traced out quite far, forming what is called a mini-halo. The existence of these features surrounding the central strong radio galaxies is well established in the Perseus and Virgo cluster. Mini-halos have a size much smaller than the cluster-wide radio halos and relics (1\%-20\%). Since the active galaxy responsible for the emission in these sources is known, problematics are different in mini-halos from halos and relics. Therefore, we will not consider this class here.

\section{Cluster-wide halos}

The prototype example of this class of sources, residing at the cluster centers, is Coma-C, belonging to the Coma cluster (A1656). Halos show an essentially regular radio morphology and no or little polarization. An up- 
per limit to the polarized flux in Coma-C is $\sim 10 \%$. Significant polarization was detected only in the highest brightness region of A2256, at the level of $\sim 20 \%$ at $20 \mathrm{~cm}$ [1]. The clusters presently known to harbor a cluster-wide radio halo are given in Table 1, where we give largest linear size, monochromatic power at $1.4 \mathrm{GHz}$, total power between $10 \mathrm{MHz}$ and $10 \mathrm{GHz}$, spectral index, minimum energy density, minimum total energy and equipartition magnetic field. Equipartition parameters were computed under the standard assumptions $(\Phi=1$ and $k=1)$, integrating the spectrum between 10 $\mathrm{MHz}$ and $10 \mathrm{GHz}$ with the proper spectral index. The last column gives the reference to recent papers.

The existence of a radio halo has been suggested in the literature for few other clusters. From new VLA data [2], we exclude the presence of a halo in A401, A754, A1609 and A2142, where the previous detection is due to discrete sources, while more sensitivity is needed to draw conclusions for $\mathrm{A} 610, \mathrm{~A} 665$ and $0016+16$.

TABLE 1. Parameters of halos

\begin{tabular}{|c|c|c|c|c|c|c|c|c|}
\hline Clus & $\begin{array}{l}\text { Size } \\
\text { kpc }\end{array}$ & $\begin{array}{l}\mathrm{P}_{1.4} \\
\mathrm{~W} \mathrm{~Hz}^{-1}\end{array}$ & $\begin{array}{l}\mathrm{P}_{t o t} \\
\text { erg s} \mathrm{s}^{-1}\end{array}$ & $\alpha$ & $\begin{array}{l}\mathbf{u}_{\min } \\
\operatorname{erg~cm}\end{array}$ & $\begin{array}{l}\mathrm{U}_{\min } \\
\text { erg }\end{array}$ & $\begin{array}{l}\mathrm{H}_{e q} \\
\mu \mathrm{G}\end{array}$ & Ref \\
\hline A1656 & 550 & $3.2 \times 10^{23}$ & $6.1 \times 10^{40}$ & 1.34 & $2.4 \times 10^{-14}$ & $5.0 \times 10^{58}$ & 0.5 & {$[3]$} \\
\hline A2163 & 600 & - & $3 \times 10^{41}$ & - & $5.4 \times 10^{-14}$ & $8.0 \times 10^{58}$ & 0.8 & {$[4]$} \\
\hline A2218 & 250 & $7.9 \times 10^{22}$ & $9.0 \times 10^{39}$ & 1.1 & $4.3 \times 10^{-14}$ & $5.3 \times 10^{57}$ & 0.7 & {$[5,2]$} \\
\hline A2255 & 725 & $2.5 \times 10^{23}$ & $1.6 \times 10^{41}$ & $\gtrsim_{1.5}$ & $3.1 \times 10^{-14}$ & $5.3 \times 10^{58}$ & 0.6 & {$[6,2]$} \\
\hline A2256 & 700 & $1.2 \times 10^{23}$ & $1.6 \times 10^{41}$ & $\widetilde{1.9}$ & $1.1 \times 10^{-14}$ & $1.1 \times 10^{58}$ & 0.5 & {$[7,8]$} \\
\hline A2319 & 660 & $5.1 \times 10^{23}$ & $9.2 \times 10^{40}$ & 1.3 & $3.7 \times 10^{-14}$ & $7.1 \times 10^{58}$ & 0.6 & {$[2]$} \\
\hline
\end{tabular}

\section{Relics}

Diffuse cluster sources, similar to the central halos, located in the cluster peripheral regions were suggested to be relics of currently inactive radio galaxies. However, no compelling evidence of this has been found so far. The prototype of this class is $1253+275$, in the Coma cluster. Moreover, the Coma cluster is permeated by diffuse radio emission of very low brightness, in the region between the central halo Coma-C and the peripheral source $1253+275$. This diffuse source is referred to as Coma bridge. The relics known so far are listed in Table 2, with their distance from the cluster center, and the parent cluster. The source parameters are in Table 3, where columns have the same meaning as in Table 1 . The properties of relic radio sources are comparable to those of central halos. They have similar 
largest size, but their morphology is generally more irregular and elongated. Moreover, in $1253+275$ and in $0917+75$, a significant percentage of polarized flux is detected. The difference in the polarization properties could be naturally explained from the larger number of magnetic field cells along the line of sight at the cluster center, compared to the outer regions.

TABLE 2. Relics

\begin{tabular}{lll}
\hline Name & Distance from c.c. & Cluster \\
\hline $0038-096$ & $320 \mathrm{kpc}$ & A85 \\
$0917+75$ & $2.5 \mathrm{Mpc}$ & A786, Rood\#27 \\
$1253+275$ & $1.35 \mathrm{Mpc}$ & A1656 \\
Coma Bridge & $1.1 \mathrm{Mpc}$ & $\mathrm{A} 1656$ \\
$1401-33$ & $90 \mathrm{kpc}$ & $\mathrm{S} 753$ \\
$2006-56$ region & $1.04 \mathrm{Mpc}$ & $\mathrm{A} 3667$ \\
\hline
\end{tabular}

TABLE 3. Parameters of relics

\begin{tabular}{|c|c|c|c|c|c|c|c|c|}
\hline Name & $\begin{array}{l}\text { Size } \\
\text { kpc }\end{array}$ & $\begin{array}{l}\mathrm{P}_{1.4} \\
\mathrm{~W} \mathrm{~Hz}^{-1}\end{array}$ & $\begin{array}{l}\mathrm{P}_{t o t} \\
\mathrm{erg} \mathrm{s}^{-1}\end{array}$ & $\alpha$ & $\begin{array}{l}\mathrm{u}_{\min } \\
\mathrm{erg} \mathrm{cm}\end{array}$ & $\begin{array}{l}\mathrm{U}_{\min } \\
\text { erg }\end{array}$ & $\begin{array}{l}\mathrm{H}_{e q} \\
\mu \mathrm{G}\end{array}$ & Ref \\
\hline 0038-096 & 200 & $5.1 \times 10^{23}$ & $1.6 \times 10^{41}$ & $>1.5$ & $1.5 \times 10^{-13}$ & $1.8 \times 10^{58}$ & 1.3 & {$[2]$} \\
\hline $0917+75$ & 780 & $2.0 \times 10^{24}$ & $1.7 \times 10^{41}$ & 1.0 & $3.5 \times 10^{-14}$ & $2.8 \times 10^{58}$ & 0.6 & {$[9]$} \\
\hline $1253+275$ & 580 & $1.7 \times 10^{23}$ & $2.0 \times 10^{40}$ & 1.1 & $3.4 \times 10^{-14}$ & $1.1 \times 10^{58}$ & 0.6 & {$[10]$} \\
\hline Coma Br. & 970 & $4.9 \times 10^{22}$ & $1.6 \times 10^{40}$ & 1.5 & $9.8 \times 10^{-15}$ & $2.2 \times 10^{58}$ & 0.3 & {$[11]$} \\
\hline $1401-33$ & 220 & $1.0 \times 10^{23}$ & $2.5 \times 10^{40}$ & 1.4 & $9.7 \times 10^{-14}$ & $6.2 \times 10^{57}$ & 1.0 & {$[12]$} \\
\hline $2006-56$ & 870 & $2.6 \times 10^{24}$ & $3.7 \times 10^{41}$ & 1.2 & $2.3 \times 10^{-14}$ & $1.5 \times 10^{58}$ & 0.5 & {$[13]$} \\
\hline
\end{tabular}

\section{Confinement}

It is evident from Tables 1 and 3 that the minimum energy densities in halos and relics are very similar. If these radio sources are in pressure equilibrium with the ambient medium, we would expect much higher energy densities in halos with respect to relics, reflecting the pressure profile of the cluster intergalactic medium. Typically, the minimum internal pressure in halos is about 3 order of magnitude lower than the external pressure of the ambient gas, obtained by X-ray data. For peripheral relics, the X-ray data are scarce. However, an imbalance of about a factor of 10 was found for $1253+275$ in 
the Coma cluster [10]. Our conclusion is that the energy density in these sources is likely to be higher than the minimum value.

\section{The Coma-C spectrum and the Coma cluster magnetic field}

The halo Coma-C is the best studied example of a cluster-wide radio halo. The spectral index distribution [3] shows a central plateau with an almost constant value $\alpha \sim 0.8$, and an outer spectral steepening, with values of $\alpha$ increasing up to 1.8 . This behaviour provides strong evidence that the particle reacceleration mechanism is more efficient at the cluster center. Since the central plateau is comparable in size to the region within one cluster core radius, the turbulent acceleration mechanism is likely to be related to wakes produced by the galaxy motions.

An important information on the magnetic field in the Coma cluster can be obtained from the rotation measure studies of the tailed radio galaxy NGC4869, located in the cluster central region and therefore seen through the intracluster medium. The polarization properties of this radio galaxy [14] indicate the presence of local fluctuations in the rotation measure (RM), occurring on typical scales of $\approx 2.5^{\prime \prime}$, i.e. $0.85 \mathrm{kpc}$, and arising in a screen external to the source. The dispersion of RM observed across NGC4869, explained by assuming that the magnetic field is tangled on typical scales of the same size as the RM fluctuations, leads to a strength of magnetic field associated with the intergalactic medium between 8.3 and $11.7 \mu \mathrm{G}$, depending on the location of the radio galaxy along the line of sight (see also [15]). Even allowing for uncertainties related with this determination, the Coma magnetic field is more than one order of magnitude larger than the equipartition value (Table 1 ).

The evidence for a magnetic field larger than the equipartition value has been suggested by [16] for the diffuse source $0917+75$ : from the absence of $\mathrm{X}$-ray synchro-self Compton emission a lower limit of $1 \mu \mathrm{G}$ was found, which should be compared with the $0.6 \mu \mathrm{G}$ given in Table 3 . These data favour the hypothesis that the energy density is not minimum, as already suggested by the confinement arguments.

\section{Properties of clusters with halos}

The properties of clusters containing radio halos and relics are presented in Table 4. X-ray data are from [17] and [18]. We remind here that the Coma cluster contains both a central halo and peripheral relics. The clusters with halos are characterized by: i) high richness, ii) no strong galaxy concentration, iii) high $\mathrm{X}$-ray luminosity, iv) no cooling flow, v) high temperature (7-14 Kev). The cooling flow in A2319 is not confirmed by recent ASCA results (Yamashita, private communication). Also, the central dominant 
galaxy in this cluster is not very dominant [19]. The fact that halos form in clusters with high temperature, i.e. large mass, is not of obvious interpretation. Moreover, high temperature and high X-ray luminosity imply a high central pressure. This fact seems in contrast with the pressure imbalance mentioned in Sect. 4, and would support the suggestion that internal pressures within halos are not minimum.

TABLE 4. Cluster properties

\begin{tabular}{llllllll}
\hline Name & $z$ & RS & BM & Rich. & $\begin{array}{l}\mathrm{L}_{x}(2-10 \mathrm{keV}) \\
\mathrm{erg} \mathrm{s}^{-1}\end{array}$ & $\begin{array}{l}\mathrm{T} \\
\mathrm{keV}\end{array}$ & Cool flow \\
\hline Coma & 0.0232 & B & II & 2 & $2.1 \times 10^{44}$ & 8.11 & NO \\
A2163 & 0.2010 & I & - & 2 & $1.8 \times 10^{45}$ & 13.9 & - \\
A2218 & 0.1710 & - & II & 4 & $2.5 \times 10^{44}$ & 6.72 & NO \\
A2255 & 0.0809 & C & II-III & 2 & $1.3 \times 10^{44}$ & 7.3 & NO \\
A2256 & 0.0601 & B & II-III & 2 & $2.9 \times 10^{44}$ & 7.51 & NO \\
A2319 & 0.0564 & cD & II-III & 1 & $4.4 \times 10^{44}$ & 9.9 & WEAK \\
& & & & & & & \\
A85 & 0.0518 & cD & I & 1 & $1.9 \times 10^{44}$ & 6.2 & YES \\
A786 & 0.1241 & F & - & 0 & - & - & - \\
S753 & 0.0139 & - & I & 0 & - & - & - \\
A3667 & 0.0552 & - & I-II & 2 & $2.6 \times 10^{44}$ & 6.5 & NO \\
\hline
\end{tabular}

From X-Ray observations of A2256, it was suggested that the presence of a halo source could be related with the existence of a cluster merger process $[20,21]$. According to $[22]$, the interaction between the intergalactic medium of a subgroup and that of the main cluster may produce shocks which can accelerate the electrons radiating in the halos, and amplify the magnetic field. The merger hypothesis is consistent with the presence of halos in clusters without a cooling flow, since a major merger event is expected to disrupt a cooling flow.

We found indeed that all the clusters in Table 1 show the evidence of a recent merger, either from X-ray or optical data. In the Coma cluster, the radio halo could be associated with the ongoing merger at the cluster center, between the two groups centered on the two dominant galaxies NGC4874 and NGC4889, close in projected distance but offset by about $800 \mathrm{~km} / \mathrm{s}$ [23]. The merger between the NGC4839 group and the main cluster, which was originally suggested to be responsible for the halo energy supply [24], is in our opinion not related with Coma-C, because it occurs in a peripheral region. Also in the case that the group has already passed through the cluster core about 2 Gyrs ago [25], it is difficult to reconcile the 
merger energy release with the spectrum of Coma-C. However, this merger could be relevant for the maintenance of the Coma bridge, and of the relic $1253+275$. Therefore, an ongoing merger process seems to be crucial for the energy supply to the radio halo, in addition to the energy possibly provided by galactic wakes (Sect. 5).

We note, however, that the merger alone cannot account for the origin of halos, as the existence of a merger process seems to be common in clusters of galaxies, while the halo sources are a rare phenomenon. For example, the cluster A754 shows a subcluster collision both from optical and X-ray data $[26,27]$, but no radio halo is detected (Sect. 2). In the case of Coma$\mathrm{C}$, it was suggested [3] that the tailed radio galaxy NGC4869, which is orbiting around the cluster center is responsible for the relativistic electron supply. The need of tailed radio galaxies residing at the cluster centers, as the origin of relativistic particles, could explain the rarity of halo type radio sources. In conclusion, the formation of a halo can be due to a cluster merger event, which amplifies magnetic fields and reaccelerates the existing particles, deposited by tailed radio galaxies. The origin of relics is more puzzling, but we point out that relics have many similarities with halos.

\section{References}

[1] Bridle, A.H., Fomalont, E.B., Miley, G.K., Valentijn, E.A. (1979) $A \& A$, 80, 201

[2] Feretti, L., Giovannini, G., L., Böhringer, H. (1995), in preparation

[3] Giovannini,G.,Feretti,L.,Venturi,T.,Kim,K.T.,Kronberg,P.P.(1993) ApJ, 406, 399

[4] Herbig, T., Birkinshaw, M. (1994) BASS, 26, 1403

[5] Partridge, R.B., Perley, R.A., Mandolesi, N., Delpino. F. (1987) ApJ, 317, 112

[6] Burns, J.O., Roettiger, K., Pinkney, J., et al. (1995) ApJ, 446, 583

[7] Kim, K.T. (1995) ApJ, Submitted

[8] Röttgering, H., Snellen, I., Miley, G., et al. (1994) ApJ, 436, 654

[9] Harris, D.E., Stern, C.P., Willis, A.G., Dewdney, P.E. (1993) $A J, 105,769$

[10] Giovannini, G., Feretti, L., Stanghellini, (1991) $A \& A, 252,528$

[11] Kim, K.T., Kronberg, P.P., Giovannini, G. Venturi, T. (1989) Nature, 341, 720

[12] Goss,W.M.,McAdam,W.B.,Wellington,K.J.,Ekers,R.D.(1987) MNRAS, 226, 979

[13] Goss, W.M., Ekers, R.D., Skellern, D.J., Smith, R.M. (1982) MNRAS, 198, 259

[14] Feretti, L., Dallacasa, D., Giovannini, G., Tagliani, A. (1995) $A \mathcal{E} A$, 302, 680

[15] Felten J.E. (1996) in Clusters, Lensing and the Future of the Universe, ed. V. Trimble, ASP Conference, in press

[16] Harris, D.E., Willis, A.G., Dewdney, P.E., Batty, J. (1995) MNRAS 273, 785

[17] David, L.P., Slyz, A., Jones, C., et al. (1993) $A p J, 412,479$

[18] Edge, A.C., Stewart, G.C., Fabian, A.C. (1992) MNRAS, 258, 177

[19] Hanisch, R.J. (1982) $A \& A, 116,137$

[20] Briel, U.G., Henry, J.P., Schwarz, R.A., et al. (1991) $A \& A, 246$, L10

[21] Fabian, A.C., Daines, S.J. (1991) MNRAS, 252, 17P

[22] Tribble, P.C. (1993) MNRAS, 263, 31

[23] Colless, M., Dunn, A.M. (1995) $A p J$, in press

[24] Briel, U.G., Henry, J.P., Böhringer, H. (1992) $A \& A$, 259, L31

[25] Burns, J.O., Roettiger, K., Ledlow, M., Klypin, A. (1994) $A p J, 427, \mathrm{~L} 87$

[26] Henry P.J. \& Briel U.G. (1995) $A p J, 443$, L9

[27] Zabludoff A.I. \& Zaritsky D. (1995) $A p J, 447$, L21 